\title{
Linx
}

Revue des linguistes de l'université Paris X Nanterre

$62-63 \mid 2010$

Entre syntaxe et discours. Éclairages

épistémologiques et descriptions linguistiques

\section{Flou phraséologique, quasi-grammaticalisation et pseudo marqueurs de discours : un no man's land entre syntaxe et discours?}

\section{Catherine Bolly}

\section{OpenEdition}

\section{Journals}

Édition électronique

URL : http://journals.openedition.org/linx/1356

DOI : $10.4000 /$ linx.1356

ISSN : 2118-9692

\section{Éditeur}

Presses universitaires de Paris Nanterre

Édition imprimée

Date de publication : 1 juillet 2010

Pagination : 11-38

ISSN : 0246-8743

\section{Référence électronique}

Catherine Bolly, «Flou phraséologique, quasi-grammaticalisation et pseudo marqueurs de discours: un no man's land entre syntaxe et discours ? », Linx [En ligne], 62-63 | 2010, mis en ligne le 25 juin 2013, consulté le 19 avril 2019. URL : http://journals.openedition.org/linx/1356 ; DOI : 10.4000/ $\operatorname{linx} .1356$ 


\title{
Flou phraséologique, quasi-grammaticalisation et pseudo marqueurs de discours : un no man's land entre syntaxe et discours?
}

\author{
Catherine Bolly \\ F.R.S.-FNRS \& Université catholique de Louvain \\ catherine.bolly@uclouvain.be
}

\section{Introduction}

Cet article propose d'étudier la nature du lien entre les deux cadres épistémologiques de la phraséologie et de la grammaticalisation qui, bien que s'ignorant encore largement à l'heure actuelle, présentent un grand nombre de caractéristiques communes: (i) opacité et non-compositionnalité en phraséologie, déplacement/blanchiment sémantique et (inter-)subjectification en grammaticalisation; (ii) fixité syntaxique en phraséologie, autonomisation syntaxique et prosodique en grammaticalisation ; (iii) collocabilité et attraction lexicale en phraséologie, coalescence syntagmatique et phonologique en grammaticalisation. Nous verrons en quoi certaines de ces caractéristiques peuvent être les indices d'un processus de constructionnalisation en cours, en particulier pour les constructions verbales à fonction pragmatique (e.a. tu vois) qui, bien qu'appartenant (encore) au domaine de la syntaxe, se comportent (déjà) comme des marqueurs de discours en français contemporain. Adoptant une vision constructionnelle de la phraséologie, l'accent sera mis dans le présent article sur la 
polyfactorialité et la gradualité, en tant que critères définitoires à la fois du processus de figement en phraséologie (perspective synchronique) et du processus évolutif en grammaticalisation (perspective diachronique).

\section{Phraséologie et collocation}

La phraséologie, dont l'origine remonte à la notion de groupes phraséologiques chez Bally (1909), connait un essor considérable depuis les années 1980 et trouve actuellement de nombreux échos en linguistique appliquée : développement de programmes d'extraction d'unités polylexicales (Blanc et al., 2008, 2010), études empiriques et psycholinguistiques sur l'acquisition d'expressions idiomatiques, de collocations ou de formules (Ellis et al., 2008 ; Forsberg, 2008 ; Conklin \& Schmitt, 2008 ; Wood, 2010), prise en compte des collocations dans l'élaboration de manuels scolaires et de dictionnaires (Granger \& Paquot, 2008a ; Tutin, 2010). De manière générale, les études menées en phraséologie durant les dernières décennies sont représentatives de deux grandes tendances (Granger \& Paquot, 2008b; Bolly, 2008, 2011): l'approche fonctionnelle, qui se centre sur l'étude des unités caractérisées par un degré minimal de figement sémantique (selon des critères linguistiques), et l'approche statistique, qui s'intéresse aux unités polylexicales caractérisées au minimum par une attraction lexicogrammaticale ente unités (selon des critères de récurrence et de cooccurrence).

\subsection{Phraséologie fonctionnelle}

Dans une perspective fonctionnelle de la phraséologie, les notions de figement, expressions idiomatiques, locutions, séquences préfabriquées ou formules (pour ne citer qu'elles) suscitent l'intérêt croissant des linguistes surtout à partir des années 1990 (e.a. G. Gross, 1996 ; Martins-Baltar, 1995, 1997 ; Fiala et al., 1997 ; Mel'čuk, 1993, 1995, 1998 ; Cowie 1998 ; Moon, 1998 ; González Rey, 2002 ; Mejri, 1997, 2005 ; Wray, 2002, 2007, 2008). À l'instar de Wray (2002, 2007), nous distinguons trois types d'unités phraséologiques : référentielles, organisationnelles et interactionnelles. Les unités référentielles ont pour fonction première la transmission d'un contenu linguistique propositionnel à valeur dénominative (par ex. : voir ronge, Dét. point de vue, à vue de nę). Les unités organisationnelles permettent d'organiser ou de structurer le discours, tant du point de vue des unités logico-sémantiques (niveau micro-syntaxique de la phrase) que de celui des unités discursives (niveau macro-syntaxique du texte). Cette catégorie comprend donc les locutions prépositionnelles et conjonctives (par ex. : étant donné (que), vu que) et les marqueurs complexes d'organisation du discours (par ex. : dans un premier/deuxième temps, voilà quor), qui peuvent émaner de structures subordonnantes avec un verbe fréquent à haut potentiel cognitif (par ex. : on a vu (précédemment) que). Les unités interactionnelles, appelées aussi pragmatèmes (Mel'čuk, 1998, p. 28-29) sont fortement dépendantes du contexte situationnel dans lequel elles apparaissent. Ces unités peuvent avoir prioritairement : (i) une fonction expressive de transmission d'un contenu extralinguistique (croyances, attitude, sentiment, etc.) (par ex. : tu rigoles ou quoi ?; tu parles.); (ii) une fonction phatique d'établissement, de maintien ou d'arrêt de la communication avec le ou les interactant(s) (par ex. : th vois ce que je veux dire) ; (iii) une fonction d'ancrage dans un cadre socioculturel ou dans un genre discursif 
particuliers (par ex. : (comment) ca va? ; Veuillez agréer, Madame, Monsieur, l'expression de mes sincères salutations). Les unités phraséologiques, pour les tenants de l'approche fonctionnelle, sont des unités polylexicales caractérisées par un certain degré de figement syntaxique, sémantique, lexical et/ou pragmatique.

\section{1) Critères de figement}

Le figement est un processus polyfactoriel (cf. Lamiroy \& Klein, 2005, p. 135) agissant à de multiples niveaux de l'analyse langagière. Deux mécanismes d'ordre cognitif, qui participent au processus dynamique de création langagière, jouent ainsi un rôle crucial dans le processus de figement des unités phraséologiques: (i) la mémorisation cognitive, i.e. l'intégration mémorielle des unités phraséologiques comme des unités sémantiquement autonomes (enregistrées en mémoire discursive as a whole); (ii) l'institutionnalisation ou conventionnalisation, i.e. la reconnaissance partagée du caractère conventionnel des unités phraséologiques par la communauté linguistique concernée. Par ailleurs, quatre critères linguistiques font l'objet d'un relatif consensus pour caractériser le figement: (i) la polylexicalité caractérise les unités complexes discontinues, autrement dit les unités correspondant à une seule catégorie grammaticale composée de plusieurs mots (lexèmes) séparés par un blanc (i.e. discontinues) (Bolly, 2011, p. 32) ; (ii) la fixité ( $v$ s. flexibilité) se traduit par une contrainte sur l'ordre des constituants et sur les propriétés transformationnelles d'une séquence donnée (e.a. passivation, pronominalisation, focalisation en c'est... que, dislocation à gauche et transformation interrogative pour les structures verbo-nominales telles que prendre position) (G. Gross, 1996 ; Mejri, 1997) ; (iii) la non-compositionnalité (vs. compositionnalité, i.e. transparency), souvent associée à la notion d'opacité (vs. transparence), est le résultat du sens «non déductif des formatifs entre eux» (González Rey, 2002, p. 57) : le signifié global de l'unité polylexicale n'équivaut pas à la somme du sens littéral de ses constituants (on parle alors de noncompositionnalité) et ne peut donc pas non plus être déduit à partir du sens de ces derniers (on parle d'inanalysabilité) ${ }^{1}$; (iv) la restriction paradigmatique (vs. ouverture des paradigmes synonymiques, i.e. commutability) renvoie aux contraintes lexicales pesant sur les possibilités de commutation par substitution synonymique des paradigmes au sein de l'unité complexe. Autrement dit, contrairement aux séquences libres qui offrent des « cases » syntaxiques non saturées lexicalement, une unité phraséologique se caractérise par une saturation lexicale au moins partielle (Mejri, 1997, p. 34-35). Le seuil minimal de figement des unités phraséologiques, selon l'approche fonctionnelle, est traditionnellement fixé à une non-compositionnalité au moins partielle avec une restriction paradigmatique forte pesant sur au moins un des deux constituants de la séquence.

\section{2) Collocations restreintes}

La phraséologie fonctionnelle envisage le phénomène du figement par opposition à la liberté combinatoire, en situant ces deux pôles sur l'axe des relations syntagmatiques entre unités linguistiques. Une séquence est ainsi dite libre (Ex. 1) quand elle ne fait l'objet d'aucune restriction idiosyncrasique : les seules contraintes

1 Nous renvoyons à Svensson (2004) pour plus de détail sur la relation que les divers critères et indicateurs sémantiques de figement entretiennent entre eux : opacité vs. transparence, non-compositionnalité vs. compositionnalité, démotivation vs. motivation, inanalysabilité vs. analysabilité, figurativité vs. non-figurativité. 
linguistiques qui régissent la combinatoire de cette séquence sont les règles de syntaxe et les règles de restriction de sélection sémantique.

(1) Ma main se tendit toute seule, prit un fruit et le porta à ma bouche. (Litt., Contemporain, corpus ComparaF - J. Harpman, Ève, 2001)

C'est ainsi que dans l'exemple (1), la séquence se caractérise par : (i) sa flexibilité : la transformation passive, l'extraction du complément postverbal et l'insertion d'un adverbe (par ex. : soudain, adroitement) entre le verbe et le syntagme nominal sont acceptables ; (ii) sa compositionnalité : le verbe prendre est actualisé dans son sens littéral ${ }^{2}$ d'acte de 'préhension physique' et le lexème fruit dans son sens littéral de 'production des plantes apparaissant après la fleur'; (iii) l'ouverture des paradigmes synonymiques: le lexème fruit est substituable par des noms quasi synonymiques (par ex. : pomme, poire) et le verbe prendre par le verbe quasi synonymique saisir.

Par contraste, une unité est dite collocationnelle quand elle présente un certain nombre de contraintes en termes de fixité, de non-compositionnalité et/ou de restriction paradigmatique, voire de contrainte situationnelle ou pragmatique. Ces contraintes se traduisent généralement par un certain degré de collocabilité mesurable statistiquement en termes de fréquence de cooccurrence des constituants (cf. phraséologie statistique, infra). La co-apparition préférentielle entre les constituants d'une collocation est imposée par l'usage au sein d'une communauté linguistique particulière : elle est dite conventionnelle. Nous considérerons par exemple la séquence prendre des nouvelles de qqn (Ex. 2) comme étant une unité phraséologique collocationnelle.

(2) Dans quelques années, disait-elle, tu verras qu'il n'y aura plus personne pour prendre de nos nouvelles. (Litt., Contemporain, corpus ComparaFP. Adam, Préface, 2003)

Cette séquence se caractérise par: (i) un certain degré de fixité: bien que les transformations syntaxiques (e.a. passivation, interrogation, extraction) ne soient pas acceptables, il y a possibilité d'insertion d'un adverbe (par ex. : régulièrement, aussi souvent) entre le verbe et le syntagme nominal ; (ii) une non-compositionnalité partielle : bien qu'il y ait maintien du sens prototypique de 'nouvelle', l'actualisation d'une acception non prototypique de prendre ( $\neq$ 'préhension physique') reflète le premier stade du processus d'abstraction sémantique de l'élément verbal ; (iii) des restrictions sur les possibilités de substitution paradigmatique : le lexème nouvelles (i.e. la base de la collocation) sélectionne le verbe prendre (i.e. le collocatif) et refuse la co-apparition avec le verbe quasi

\footnotetext{
2 Si l'on en croit Rastier, il faut distinguer le sens de la signification: «La signification est un type, constitué comme tel par le linguiste à partir des sens observés dans le discours, qui ont le statut d'occurrences» (Rastier et al., 1994, p. 34). Or, en tant que type, le verbe prendre s'inscrit dans le schéma cognitif de l'établissement d'une relation de saisie par un sujet/agent sur un objet/patient. À l'emploi significationnel de prendre (i.e. le type) correspondent ainsi une série de caractéristiques sémantiques cognitives typiques (à savoir les traits inbérents de préhension concrète, de mouvement spatial centripète, d'action consciente et de mise en présence), liées à l'inscription du geste de préhension concrète dans un cadre spatio-temporel situé (cf. Bolly, 2011). Ce sont ces traits « hérités par défaut du type dans l'occurrence, si le contexte n'y contredit pas » (Rastier, 2005), qui déterminent le degré de littéralité du sens de l'occurrence en contexte.
} 
synonymique saisir; il y a figement sur le morphème du pluriel du nom nouvelles (nonacceptabilité du singulier). Les collocations (par ex. : critique virulente, parler ouvertement, prendre vie) se définissent donc comme des séquences polylexicales dont les constituants entretiennent une relation lexicalement contrainte, tout en conservant leur caractère compositionnel et leur contenu catégoriel ${ }^{3}$ propres. On parle alors de cooccurrence lexicale restreinte, autrement dit d'une cooccurrence lexicale plus ou moins immédiate 4 qui se caractérise par le caractère arbitraire, c'est-à-dire non prédictible et conventionnel, de co-apparition de ses constituants. Dans sa définition fonctionnelle (vs. statistique), la collocation se compose d'une base et d'un collocatif, le collocatif étant sémantiquement contraint et sélectionné par la base. Par exemple dans feu rouge, rouge est la base de la collocation qui sélectionne le sens de 'feu de la circulation' (cf. François \& Manguin, 2006, p. 52).

\subsection{Phraséologie statistique}

La phraséologie statistique, héritière de la linguistique de corpus anglo-saxonne (Sinclair, 1991, 2000 ; Römer, 2009), repose sur la reconnaissance de deux principes organisateurs du langage : le principe d'idiomaticité (idiom principle) et le principe de libre choix (open choice principle) (Erman \& Warren, 2000). Le premier principe de choix mutuel (autrement dit, de collocabilité) illustre la tendance des unités linguistiques à coapparaître de manière arbitraire dans l'usage, telle que « the choice of one word affects the choice of others in its vicinity » (Sinclair, 1991, p. 173), alors que le second principe illustre l'idée d'une combinatoire virtuellement libre où les éléments linguistiques seraient susceptibles de co-apparaitre les uns avec les autres sans opérer de choix lexical préférentiel. Prenant appui sur le critère de fréquence collocationnelle (Sinclair, 1991), la phraséologie statistique inclut dans son champ d'étude les unités polylexicales les moins contraintes, repérables grâce à leur fréquence de cooccurrence ${ }^{5}$. La fréquence est, dans ce contexte, reconnue comme un indice extrêmement révélateur du figement langagier, tant du point de vue des affinités catégorielles et des relations syntaxiques qu'entretiennent les termes en présence (règles syntaxiques et structure valencielle inhérentes aux constituants des unités phraséologiques), que du point de vue des affinités lexico-sémantiques de ces termes (sélection sémique, traits sémantiques, polysémie, métaphore/métonymie).

\footnotetext{
3 Tel que Mejri le définit (2005, p. 189), le contenu catégoriel « renvoie à l’appartenance de toute unité à une partie du discours». Dans le cas des constructions collocationnelles verbo-nominales, chaque constituant possède un contenu catégoriel spécifique (syntaxe interne), mais c'est le verbe qui détermine le comportement syntaxique de l'unité collocationnelle dans son ensemble (syntaxe externe).

${ }^{4}$ L'immédiateté (ou contiguité) renvoie à la notion de proximité des co-constituants dans leur environnement cotextuel.

${ }^{5}$ Ce qui la différencie d'ailleurs des études en phraséologie fonctionnelle qui prennent comme objet d'étude les unités polylexicales les plus figées, dans une perspective avant tout sémantique et selon une méthode le plus souvent intuitive.
} 
Catherine Bolly

\section{1) De la cooccurrence à la collocation}

Pour les définir succinctement, les cooccurrences sont des combinaisons de deux mots graphiques (tokens) caractérisés par une proximité cotextuelle et qui coapparaissent de manière fréquente dans l'usage. On parle alors de récurrence. Par extension, une collocation est une cooccurrence statistiquement significative, i.e. une séquence dont la co-apparition des termes ne semble pas être due au hasard: ses constituants entretiennent une relation lexicale statistiquement contrainte qui est mesurée en termes d'association lexicale. La mesure d'association lexicale (Stubbs, 1995) repose alors sur le calcul de la fréquence (observée et attendue) des termes pris isolément et de leur fréquence de co-apparition (observée et attendue), tenant compte de la longueur des textes où ils co-apparaissent. Par exemple, la construction je ne sais pas (Ex. 3) se caractérise par une haute fréquence en français parlé $(955 \mathrm{occ}$./ million de mots dans le corpus $V$ alibel) et par un indice d'association lexicale très élevé $(\mathrm{IM}=17,4)^{6}$.

(3) $[\ldots]$ certains professeurs parlent bien français (silence) et puis même les les gens qui sont appelés à / parler dans leur métier je ne sais pas / les avocats (bruit de micro) euh // etc. (Oral, Contemporain, 1991, Valibel, corpus Insécurité linguistique)

Par comparaison, son correspondant à polarité positive je sais (Ex. 4) se caractérise aussi par une fréquence élevée (1035 occ./ million de mots), mais avec un indice d'association lexicale moindre ( $\mathrm{IM}=4,4)$.

(4) $[\ldots]$ oui // ok / elle est elle fait aide familiale donc c'est son boulot c'est ça consiste en quoi alors je sais ça n'a rien à voir mais / c'est parce que tu me dis oui ça j'aime bien (Oral, Contemporain, 1991, Valibel, corpus Insécurité linguistique)

Contrairement à la définition fonctionnelle des collocations, dans une collocation au sens statistique du terme «le statut sémantique des coconstituants ne fait pas l'objet d'hypothèses a priori, on se contente d'observer une cooccurrence préférentielle » (François \& Manguin, 2006: 51). Les collocations statistiques se distinguent en cela des collocations restreintes (cf. supra) qui se caractérisent par une relation orientée et asymétrique avec restriction paradigmatique forte, où une base sélectionne un collocatif. Elles se distinguent en outre par leur inscription dans « un continuum de figement syntaxique et d'accession au statut de polylexème » et par le fait que «deux mots peuvent entretenir une collocation à distance » (Ibidem). La collocation grammaticale (vs. lexicale) désigne en particulier une combinaison lexicalement contrainte constituée d'un mot lexical (de contenu) qui sélectionne de manière arbitraire un mot

\footnotetext{
${ }^{6}$ L'indice d'information mutuelle (noté IM) est un indice statistique d'attraction entre unités lexicales (McEnery \& Wilson, 1996, p. 86). Un indice d'IM négatif ou proche de zéro indique que la coapparition des unités lexicales semble être due au hasard : leur combinatoire est relativement libre. Inversement, plus une cooccurrence lexicale a un indice d'IM élevé, plus la séquence est collocationnelle.
} 
Flou phraséologique, quasi-grammaticalisation et pseudo marqueurs de discours...

grammatical (mot-outil), généralement une préposition, ou une structure syntaxique, par exemple une proposition infinitive (par ex. : dépendre de (qqch.), assister $\underline{a}$ (qqch.); un argument contre/pour; une contribution $\underline{a}$; différent $\underline{\text { de }}$, fâché contre $\left./ \underline{\text { sur }}^{7}\right)$.

\section{2) Combinaisons syntagmatiques récurrentes}

Dans sa conception large, la phraséologie s'intéresse aux différentes combinaisons syntagmatiques réccurrentes (Bolly, 2008, 2011, p. 54-63) repérables dans l'usage par le biais d'analyses de fréquence inductives en linguistique de corpus (data driven corpus approach) :

(i) les séquences récurrentes ${ }^{8}$ (Altenberg, 1998 ; De Cock, 2003, 2004) sont constituées de plusieurs formes lexicales, non nécessairement liées catégoriellement, mais nécessairement contiguës et répétées sous une forme identique (par ex. : le trigramme ily $a^{9}$ );

(ii) la prosodie sémantique désigne des combinaisons préférentielles entre une forme lexicale et un groupe d'unités lexicales à connotation spécifique ou appartenant à un champ sémantique particulier (Hoey, 2000) (par ex. : le verbe commettre co-apparaît de manière préférentielle avec des lexèmes à connotation négative tels que crime, meurtre, faute, etc.) ;

(iii) la colligation implique, dans son acception première héritée de Firth (1957), l'identification de relations entre catégories grammaticales (par ex. : verbe + préposition ou adjectif + nom); dans son acception plus répandue, la colligation implique une relation conventionnelle entre une forme lexicale et un trait syntaxique ou une forme grammaticale en particulier; de manière générale, la colligation détermine le type de relation grammaticale que des unités linguistiques entretiennent de manière préférentielle entre elles (par ex. : la structure colligationnelle $[\mathrm{Il}+$ (Pron. pers) + semble + Adj. $+q u e]$ tend à donner des énoncés lexicalement contraints tels que il (nous, me) semble évident/clair que) ;

(iv) les patrons (ou patterns) se caractérisent par une attraction mutuelle entre des structures syntaxiques et des formes lexicales (e.a. entre une forme lexicale particulière et les prépositions, les syntagmes ou les propositions qui la suivent); ces patrons permettent de révéler des relations sémantiques particulières (cf. Hunston \& Francis, 2000) par l'observation du contexte d'apparition des items lexicaux étudiés (par ex. : en anglais, to leave apparait à l'infinitif après to decide, mais sous la forme en -ing après to hate) ;

\footnotetext{
${ }^{7}$ Cette construction, dans laquelle fâché co-apparaît avec la préposition sur, est propre à la variété du français de Belgique.

${ }^{8}$ Ces séquences récurrentes sont parfois appelées segments répétés (Habert et al., 1997) et, en anglais, clusters (Scott, 2004-2007) ou lexical bundles (Biber et al., 2004 ; Hyland, 2008).

${ }^{9}$ On compte 1551 occurrences de ce trigramme dans le corpus ComparaF (corpus équilibré comptant 1 million de mots, en français de France et de Belgique).
} 
(v) les cadres collocationnels (collocational frameworks) (Sinclair \& Renouf, 1991) se définissent comme des séquences discontinues de paires d'unités lexicales cooccurrentes prédéfinies, séparées l'une de l'autre par un mot graphique non défini à l'avance (par ex. : les paires de mots grammaticaux fréquents $[a+?+o f],[t o o+?+$ to $]$ ou $[$ many $+?+o f]) ;$ dans un cadre collocationnel, la structure de départ conditionne fortement le choix du collocatif, i.e. du terme central s'insérant dans la structure prédéfinie (par ex. : a lot of, a kind of; an act of, an example off ;

(vi) en analyse collostructionnelle (Gries \& Stefanowitsch, 2004, p. 209), la collostruction (mot-valise de l'anglais : collocation + construction) est la combinaison de ces deux types de sélection lexico-grammaticale, allant du lexème vers la structure et de la structure vers le lexème (sélection bidirectionnelle); les lexèmes qui sont sélectionnés par des structures particulières y sont appelés les collexèmes et, inversement, les structures qui sont sélectionnées par un lexème sont appelées les collostructs; sur la base de ce principe, on peut observer quels sont les collexèmes les plus significatifs pour une construction donnée (par ex. : les collexèmes montrant la plus forte attraction collostructionnelle dans les constructions [ $\mathrm{N}$ waiting to happen] sont accident, disaster et welcome).

Comme nous le verrons plus loin, certaines de ces combinaisons syntagmatiques récurrentes (en particulier les colligations, les patrons, les cadres collocationnels et les collostructions) sont à rapprocher de la notion de constructions, puisqu'elles se caractérisent par une co-sélection entre une unité lexicale (ou un groupe d'unités lexicales) et les structures syntaxiques dans lesquelles elle(s) s’insère(nt) (Francis, 1993).

\section{Grammaticalisation et pragmaticalisation}

Dans son acception générale, la grammaticalisation désigne un mouvement diachronique qui voit certaines unités lexicales acquérir progressivement une fonction grammaticale (par ex. : le nom latin homo 'être humain', qui a donné lieu au pronom impersonnel on) ou certaines unités grammaticales acquérir une fonction davantage grammaticale (par ex.: le verbe de mouvement aller, qui a donné lieu au futur périphrastique). En tant que processus relevant du changement linguistique et, plus particulièrement, du champ de la grammaticalisation, la pragmaticalisation (Erman \& Kotsinas, 1993 ; Dostie, 2004) se définit quant à elle comme un mouvement évolutif qui voit des unités lexicales migrer, au cours des siècles, vers le domaine pragmatique du discours (par ex. : le verbe savoir, qui a donné lieu au marqueur discursif t'sais). Audelà du champ théorique que le terme recouvre, la grammaticalisation (MarchelloNizia, 2006) renvoie à un processus évolutif que l'on peut caractériser selon plusieurs principes et paramètres. Alors que les principes (Hopper, 1991) mettent en évidence le rôle du contexte dans l'émergence et dans l'évolution de certaines formes linguistiques, les paramètres visent à décrire quels sont les mécanismes linguistiques en jeu dans le processus lui-même (Lehmann, 1995). 
Flou phraséologique, quasi-grammaticalisation et pseudo marqueurs de discours...

\subsection{Paramètres}

Partant du constat que les unités en cours de grammaticalisation subissent une perte d'autonomie formelle et sémantique, Lehmann (1995) met en exergue une série de paramètres qui permettent de préciser quel serait le degré de grammaticalisation de ces unités. Six paramètres sont ainsi répertoriés selon que le type de changement porte sur l'axe paradigmatique ou syntagmatique de la langue, et que le changement opère au niveau du poids, de la cohésion et de la variabilité des unités linguistiques concernées (cf. Tableau 1).

\begin{tabular}{|l|l|l|}
\hline & \multicolumn{1}{|c|}{ Axe paradigmatique } & \multicolumn{1}{c|}{ Axe syntagmatique } \\
\hline $\begin{array}{l}\text { Poids } \\
\text { (weight })\end{array}$ & $\begin{array}{l}\text { Perte d'intégrité (integrity) } \\
\text { (désémantisation, attrition) }\end{array}$ & $\begin{array}{l}\text { Réduction de la portée } \\
\text { syntagmatique (structural } \\
\text { scope) (condensation) }\end{array}$ \\
\hline $\begin{array}{l}\text { Cohésion } \\
\text { (cohesion) }\end{array}$ & $\begin{array}{l}\text { Augmentation de la cohésion } \\
\text { paradigmatique (paradigmaticity) } \\
\text { (intégration paradigmatique) }\end{array}$ & $\begin{array}{l}\text { Augmentation de la } \\
\text { cohésion syntagmatique } \\
\text { (bondedness) (coalescence) }\end{array}$ \\
\hline $\begin{array}{l}\text { Variabilité } \\
\text { (variability) })\end{array}$ & $\begin{array}{l}\text { Réduction de la variabilité inter- et } \\
\text { intra-paradigmatique (caractère } \\
\text { obligatoire, obligatoriness) }\end{array}$ & $\begin{array}{l}\text { Réduction de la variabilité } \\
\text { syntagmatique (fixation } \\
\text { syntaxique ou } \\
\text { rigidification) }\end{array}$ \\
\hline
\end{tabular}

Tableau 1. Paramètres de Lehmann, à partir de De Mulder (2001, p. 11)

Premièrement, le poids d'un signe linguistique est «la propriété par laquelle le signe peut se distinguer des autres membres de sa classe et acquérir une certaine prééminence dans le syntagme: plus un signe a de poids, plus il est autonome» (De Mulder, 2001, p. 11). Un signe linguistique perd donc du poids quand ses frontières deviennent moins nettes et qu'il perd tout ou partie de son autonomie phonétique et accentuelle : ce signe s'oppose dès lors moins fortement aux autres signes du système de la langue. Sur l'axe paradigmatique, son intégrité substantielle devient plus faible, ce qui se traduit à la fois par une perte de "substance sémantique » (i.e. une désémantisation) et une perte de "substance phonologique» (i.e. une attrition) : "Un signe possède une 'substance' qui lui permet de garder son identité et de s'opposer à d'autres signes ; plus il est grammaticalisé, plus il perd de cette 'substance'» (De Mulder, 2001, p. 12). Sur l'axe syntagmatique, on observe que la portée du signe se réduit au fur et à mesure qu'il se grammaticalise, comme c'est le cas notamment du pronom démonstratif ille qui passe d'une catégorie syntaxique supérieure de syntagme nominal (avec une fonction de définitude du référent) à une catégorie syntaxique inférieure (avec une fonction de marquage catégoriel du nom), en s'intégrant de plus en plus au syntagme nominal (pron. dém. anaph. $>$ adnominal $>$ art. déf. $>$ marque catégorielle).

Deuxièmement, la perte d'autonomie est corrélée à une augmentation de la cohésion, sachant que la cohésion renvoie aux relations systématiques et aux contraintes lexico-grammaticales que le signe en cours de grammaticalisation entretient avec les autres signes linguistiques. Sur l'axe paradigmatique, on parle d'une augmentation de la paradigmaticité. Il y a alors intégration paradigmatique de l'élément grammaticalisé dans une classe paradigmatique, avec acquisition des traits syntaxiques et sémantiques 
spécifiques à la classe en question et suppression graduelle des différences entre les membres du paradigme. C'est ainsi que les constructions avec [tu+vois] et [tu+sais] qui sont formées à partir d'éléments lexicaux différents (perception visuelle vs. cognition), voient leurs différences se réduire au fur et à mesure que le processus de grammaticalisation progresse, pour finalement s'intégrer au paradigme de la classe des constructions parenthétiques (Urmson, 1952 ; Schneider, 2007) à fonction pragmatique interpersonnelle, partageant un certain nombre de caractéristiques attribuées aux marqueurs de discours (e.a. l'optionalité et la mobilité syntaxique, le renforcement pragmatique et la désémantisation).

(5) $[\ldots]$ mais tu sais il a une couleur de viande qui est qui / est un peu c'est-àdire que il ne // c'est comme un peu en France sa viande quoi tu vois / elle n'a pas du tout la même couleur rose dont les gens ont l'habitude et / et tu sais euh bien tendre etcetera / c'est vraiment le morceau / le morceau brut / et il a de la bonne viande par exemple il a de l'excellent veau / moi je vais toujours chercher mon veau là-bas (Oral, Contemporain, 2004, Valibel, corpus Enquête PFC à Liège, locuteur blaNB1)

Sur l'axe syntagmatique, les unités qui se grammaticalisent montrent une cohésion syntagmatique (i.e. un degré de coalescence) de plus en plus forte qui se traduit par une combinatoire davantage contrainte entre les signes linguistiques en co-présence.

Troisièmement, Lehmann propose de prendre en compte la réduction de la variabilité qui signale une perte de flexibilité du signe en cours de grammaticalisation: les possibilités combinatoires du signe, en termes de mobilité et de co-apparition lexicale avec d'autres unités linguistiques, sont désormais restreintes (Marchello-Nizia, 2006, p. 49). Sur l'axe paradigmatique, on distingue ainsi deux types de restrictions en termes de variabilité : (i) la réduction de la variabilité intraparadigmatique, qui renvoie au caractère obligatoire du lexème, se traduit par une augmentation des contraintes sur les possibilités de substitution à l'intérieur d'un même paradigme ; (ii) la réduction interparadigmatique renvoie au caractère obligatoire du paradigme en lui-même (par exemple, l'emploi du pronom personnel sujet est obligatoire en français moderne, mais pas en italien). Ces deux types de variabilité paradigmatique reposent sur l'idée que «la grammaire peut imposer de façon plus ou moins stricte l'expression de la catégorie grammaticale associée aux membres du paradigme et rendre obligatoire l'emploi du signe (Lehmann parle de la nature obligatoire ou obligatoriness du signe). En général, plus un signe est grammaticalisé, moins le locuteur peut décider lui-même de l'exprimer ou pas» (De Mulder, 2001, p. 13). Sur l'axe syntagmatique, la réduction de la variabilité concerne la mobilité moindre du signe en cours de grammaticalisation, qui tend de plus en plus à occuper une position syntaxique fixe. On parle alors d'un effet de fixation syntaxique (Lehmann, 1995) ou de rigidification (Croft, 1990, cité dans De Mulder, 2001, p. 14).

Il est intéressant de noter que les pragmaticalisations, si elles partagent la plupart des traits paramétriques attribués par Lehmann aux grammaticalisations, s'en distinguent cependant par le fait qu'elles se caractérisent par un élargissement de leur portée syntaxique plutôt que par une réduction de celle-ci. La plupart du temps, l'évolution des marqueurs de discours qui se sont pragmaticalisés est par ailleurs corrélée à un processus sémantique d'(inter)subjectification (Traugott, 1997, 2010). 
Flou phraséologique, quasi-grammaticalisation et pseudo marqueurs de discours...

[Subjectification is specifically a] gradient phenomenon, whereby forms and constructions that at first express primarily concrete, lexical, and objective meanings come through repeated use in local syntactic contexts to serve increasingly abstract, pragmatic, interpersonal, and speaker-based functions. (Traugott, 1997, p. 32)

Ayant au départ une fonction propositionnelle, ces marqueurs acquerraient une fonction expressive (subjective ou intersubjective), en passant parfois par une fonction textuelle d'organisation du discours (Traugott, 1982 ; Erman \& Kotsinas, 1993).

\subsection{Principes et stades : le rôle du contexte}

Le processus de grammaticalisation est conçu comme un processus au cours duquel une entité linguistique acquiert une fonction grammaticale (ou pragmatique) dans un contexte morphosyntaxique et pragmatique particulier (Heine, 2002). Le contexte linguistique (ou cotexte) joue donc un rôle crucial dans l'émergence et dans l'évolution des formes linguistiques en cours de grammaticalisation. C'est la diversification des contextes dans lesquels l'unité linguistique apparaît qui permet l'élargissement même des possibilités sémantico-pragmatiques de l'unité en question.

In recent grammaticalization studies, it has been shown that a new grammatical function does not arise homogeneously in all uses of the linguistic item concerned, but is bound in its origin to specific linguistic 'contexts' or 'constructions'. (Diewald, $2006: 3)$

On peut ainsi voir dans les principes mis au jour par Hopper (1991) les prémices de l'articulation en stades proposée par Heine (1993, 2002). Selon Hopper (1991), cinq principes caractérisent le processus de grammaticalisation :

(i) la concurrence (layering), i.e. la coexistence de «couches» de plusieurs unités linguistiques concurrentes plus ou moins grammaticalisées à un même stade de l'évolution langagière ;

(ii) la divergence, i.e. la coexistence d'emplois (plus) grammaticalisés et d'emplois peu ou pas grammaticalisés pour une seule et même unité ;

(iii) la spécialisation fonctionnelle de l'unité qui se grammaticalise et n'apparaît plus dès lors que dans certains contextes ;

(iv) la persistance de certains traits sémantiques originels dans les emplois grammaticalisés ;

(v) la décatégorialisation, i.e. la perte des règles syntaxiques et sémantiques propres à la catégorie syntaxique d'origine de la forme, corrélée à une appropriation du comportement syntaxique et sémantique de la nouvelle catégorie.

Selon Heine, qui avait d'abord proposé une représentation en trois phases (Heine, 1993), le processus de grammaticalisation se ferait en quatre phases susceptibles de coexister durant une même période (Heine, 2002). Au stade initial, le mot susceptible de se grammaticaliser serait employé dans son sens originel dans tous ses emplois. Au second stade, qui correspond au contexte de transition (bridging context), le mot apparaitrait dans un nouveau contexte qui permettrait à son tour l'émergence d'un nouveau sens. 
Ce second stade se caractérise par la coexistence de plusieurs réalisations sémantiques concurrentes d'une même unité dans un contexte nouveau, dès lors ambigu. Autrement dit, les contextes ambigus permettraient l'apparition de sens nouveaux: "C'est donc d'abord par la modification des contextes dans lesquels le mot se rencontre que le changement apparaît» (Marchello-Nizia, 2006, p. 23). Au troisième stade, appelé contexte de passage (switch context), la nouvelle signification apparait comme étant incompatible avec le sens initial dans certains contextes (relégué à l'arrière-plan, mais toujours accessible). Le nouveau sens permettrait à son tour de créer de nouveaux contextes, cette fois non ambigus. Au quatrième et dernier stade de conventionnalisation, l'usage ancien est encore présent, mais "n'est plus senti comme ayant aucune parenté avec le nouveau morphème » (Marchello-Nizia, 2006, p. 22). Il y a primauté du sens nouveau sur l'ancienne signification.

Bien que critiquables à plusieurs égards (Béguelin, 2009, 2010) et notamment du point de vue du principe de l'unidirectionnalité (e.a. Campbell, 2001 ; Newmeyer, 2001 ; Prévost, 2003 ; Norde, 2010), nous verrons que les paramètres et principes attribués au processus de grammaticalisation restent des outils efficaces pour décrire l'évolution de certaines unités linguistiques (voir à ce propos Traugott, 2001 ; Haspelmath, 2004 ; Prévost, 2006).

\section{Phraséologie et grammaticalisation : si loin, si proche}

Après avoir décrit les deux cadres théoriques de manière isolée, nous présentons une synthèse des principaux traits définitoires attribués conjointement (bien que de manière non strictement équivalente et sous une terminologie différente) aux unités grammaticalisées et aux unités phraséologiques :

- le caractère obligatoire se définit par opposition à une combinatoire virtuellement libre et prédictible et se traduit en termes de contraintes sur la combinatoire lexico-grammaticale : réduction de la variabilité intraparadigmatique et syntagmatique (en grammaticalisation); restrictions paradigmatiques et rigidité syntaxique $^{10}$ (en phraséologie) ;

- $\quad$ le caractère progressif se traduit en termes de gradualité : évolution progressive par stades (en anglais, gradualness) (en grammaticalisation); degrés de figement (en anglais, gradience) du plus libre au plus figé, en passant par la collocation (en phraséologie) ;

- le déplacement sémantique par abstraction va souvent de pair avec un processus d'(inter)subjectification (objectif > subjectif > subjectif) et/ou de métaphorisationmétonymisation (en grammaticalisation), qui peut aboutir à la non-compositionnalité et à l'opacité des unités complexes en synchronie (en phraséologie) sur lesquelles s'exerce un mécanisme dit de coercition: «la construction exerce

\footnotetext{
${ }^{10}$ La rigidité syntaxique (vs. mobilite) renvoie aux contraintes sur l'ordre d'apparition de l'unité figée dans la chaîne syntagmatique langagière. La fixité à proprement parler correspond davantage au paramètre de réduction de la portée syntaxique en grammaticalisation, puisqu'elle concerne les contraintes sur les possibilités de transformation syntaxique (cf. supra).
} 
Flou phraséologique, quasi-grammaticalisation et pseudo marqueurs de discours...

une pression sur le lexème, pour que celui-ci devienne sémantiquement compatible avec le tout » (Legallois \& Gréa, 2007);

- la décatégorisation morphologique s'accompagne d'un mécanisme de réanalyse ${ }^{11}$ consistant en une sorte de « reparenthésage » des éléments sur lesquels porte le changement (Marchello-Nizia, 2006 : 43) et aboutit à une recatégorisation; Lehmann (1995) parle de dégénérescence morphologique pour désigner le changement des catégories majeures (verbes, noms, adjectifs) vers une catégorie mineure (adpositions, conjonctions, adverbes, auxiliaires, affixes verbaux) ; l'apparition de ces unités dans de nouveaux contextes rend possible l'émergence et la généralisation de sens nouveaux (cf. supra le rôle du contexte) ; en phraséologie, la dimension lexico-grammaticale des unités phraséologiques, dont la structure valencielle est en quelque sorte figée, reflète ce mécanisme de " reparenthésage » des frontières catégorielles ;

en grammaticalisation, la coalescence ${ }^{12}$ aboutit le plus souvent à une soudure graphique ou du moins à une contiguïté formelle, selon le chemin cohésion syntagmatique $>$ coalescence $>$ cliticisation $>$ agglutination (d'après Lehmann); ce processus aboutit à une paradigmatisation : «le nouveau morphème entre dans un paradigme existant et s'y adapte, modelant sa forme et ses constructions en conséquence » (Marchello-Nizia, 2006 : 41) ; en phraséologie, on parlera alors plus volontiers de collocabilité ou d'attraction lexicale, pour désigner cette co-apparition préférentielle entre unités lexicales ou catégories grammaticales qui peut aller jusqu’à la fusion des signes linguistiques en cooccurrence (par exemple, écoute donc a donné lieu à coudon en français québécois - cf. Dostie, 2004).

S'il est évident que la phraséologie et la grammaticalisation visent à répondre à des questions de recherche différentes et ne sont donc pas superposables de manière stricte, il n'en reste pas moins que l'étude des mécanismes langagiers qui leur sont connexes apporte un éclairage nouveau sur le fonctionnement de certaines unités linguistiques difficilement catégorisables a priori dans une catégorie linguistique précise (comme c'est notamment le cas les constructions parenthétiques). C'est ce que nous montrerons dans les pages qui suivent en nous appuyant successivement sur les notions de phraséologie constructionnelle et de grammaticalisation constructionnelle.

\footnotetext{
11 De manière succincte, la réanalyse peut se définir comme un mécanisme du changement langagier qui modifie la structure sous-jacente (underlying structure) d'une construction syntaxique, sans en modifier la forme (surface manifestation). L'importance accordée par les linguistes au mécanisme de réanalyse dans le changement langagier diffère selon les auteurs: «Most agree that grammaticalization is not a mechanism of change in its own right, but relies primarily on reanalysis (and also extension)» (Campbell, 2001 : 141).

12 L'affaiblissement phonologique observé en grammaticalisation (phonological attrition chez Lehmann, 1995 ou phonological erosion chez Heine, 2003), prosodique et accentuel (unité lexicale $>$ morphème $>$ clitique $>$ affixe $>$ marque zéro) est pour sa part encore peu pris en compte en phraséologie, souvent considéré comme un effet collatéral du processus de coalescence.
} 


\section{Phraséologie constructionnelle (approche synchronique)}

Dans le domaine actuellement en plein essor des grammaires de construction (Construction Grammar, désormais notée $\mathrm{CxG}$ ), notre approche se situe du côté des théories dites constructionnelles ( $v$ s. non constructionnelles) qui récusent l'autonomie de la syntaxe par rapport aux autres niveaux langagiers (François, 2008, p. 3). Ces théories s'inscrivent dans une approche de la grammaire plus inductive et contextualiste: elles laissent une place aux études sur corpus dites «écologiques», qui prennent en considération les activités et les contextes situationnels dans lesquels l'acte de communication langagière s'inscrit, et accordent une place importante à la dimension pragmatico-sémantique du langage, en ce que celle-ci interagit avec la syntaxe et le lexique. Par ailleurs, ces théories postulent la préexistence de mécanismes cognitifs généraux et conçoivent le langage dans une perspective émergentiste plutôt que représentationnelle : «le langage est envisagé comme instrument de conceptualisation active du monde et/ou comme instrument de communication» (Fuchs, 2004, p. 7). Nous définirons ici brièvement les constructions comme étant :

- $\quad$ un appariement entre forme et fonction/sens,

- à concevoir de manière graduelle sur un continuum entre lexique et syntaxe, par opposition à une vision modulaire du système linguistique,

- $\quad$ dans une perspective où la syntaxe est généralement motivée par des schèmes conceptuels, dans le cas des linguistiques cognitives (e.a. Langacker, 2000), et/ou par des contraintes pragmatiques, dans le cas des linguistiques néofonctionnalistes ou des grammaires émergentes (e.a. Hopper, 1988, 1998 ; Bybee, 2003 ; Goldberg, 2006).

Si l'on compare les catégories d'unités répertoriées en phraséologie et en $\mathrm{CxG}$ (cf. Tableau 2), on voit que les unités complexes discontinues (i.e. séparées par un blanc) ${ }^{13}$ regroupent à la fois les constructions complexes (formelles et substantielles) de la $\mathrm{CxG}$ et les unités phraséologiques (colligations, patrons, cadres collocationnels, collostructions, collocations et séquences figées). En dehors de ces catégories de constructions, on dépasse le champ de la phraséologie pour toucher au domaine des unités complexes continues (i.e. reliées par un trait d'union, une apostrophe ou soudées) en morphologie et en morphosyntaxe, appelées constructions complexes morphologiques en $\mathrm{CxG}$. À côté des unités complexes et des unités simples se trouvent les constructions atomiques formelles en $\mathrm{CxG}$, i.e. les catégories syntaxiques abstraites, et les constructions atomiques substantielles en $\mathrm{CxG}$, i.e. les unités monolexicales non construites.

${ }^{13}$ Cette définition de la polylexicalité a été adoptée dans le cadre de recherches menées sur l'écrit (Bolly, 2008, 2011). La définition de Meillet, selon laquelle « un mot est défini par l'association d'un sens donné à un ensemble donné de sons susceptible d'un emploi grammatical donné » (1913:30) s'est avérée inappropriée pour pouvoir effectuer les analyses sur corpus dans le cadre précis de ces recherches. Nous sommes néanmoins consciente qu'une telle définition gagnerait à être repensée en termes de polymorphémie (vs. polylexicalitê) dans le cadre d'études menées sur l'oral. 
Flou phraséologique, quasi-grammaticalisation et pseudo marqueurs de discours...

\begin{tabular}{|c|c|c|c|}
\hline & $\mathrm{CxG}$ & $\begin{array}{c}\text { Phraséologie } \\
\text { Lexique-grammaire }\end{array}$ & Exemples \\
\hline \multirow{2}{*}{ 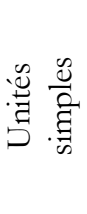 } & $\begin{array}{l}\text { Constructions } \\
\text { atomiques formelles }\end{array}$ & $\begin{array}{l}\text { Catégories syntaxiques simples } \\
\text { [Syntaxe] }\end{array}$ & $\begin{array}{l}\text { Nom, Adjectif, } \\
\text { Verbe }\end{array}$ \\
\hline & $\begin{array}{l}\text { Constructions } \\
\text { atomiques } \\
\text { substantielles }\end{array}$ & $\begin{array}{l}\text { Unités monolexémiques et } \\
\text { monomorphémiques [Lexique] }\end{array}$ & lune, petit, travail \\
\hline \multirow{3}{*}{ 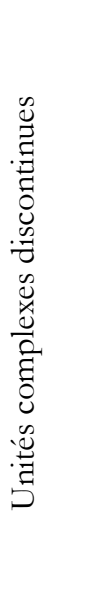 } & $\begin{array}{l}\text { Constructions } \\
\text { complexes } \\
\text { formelles }\end{array}$ & Valence [Syntaxe] & $\begin{array}{l}\text { Sujet Verbe Objet } \\
\text { Dét. Nom Adj. }\end{array}$ \\
\hline & $\begin{array}{l}\text { Constructions } \\
\text { complexes } \\
\text { substantielles } \\
\text { verbales } \\
\text { (non } \\
\text { conventionnelles) }\end{array}$ & $\begin{array}{l}\text { Combinaisons syntagmatiques } \\
\text { récurrentes : colligations, patrons, } \\
\text { cadres collocationnels et } \\
\text { collostructions [Phraséologie] } \\
\text { Cadres de sous-catégorisation } \\
\text { [Syntaxe] }\end{array}$ & $\begin{array}{l}\text { Sujet commettre Objet } \\
\text { Dét. critique Adj. }\end{array}$ \\
\hline & $\begin{array}{l}\text { Constructions } \\
\text { complexes } \\
\text { substantielles } \\
\text { verbales } \\
\text { (conventionnelles) }\end{array}$ & $\begin{array}{l}\text { Collocations restreintes, séqu. } \\
\text { figées [Phraséologie] }\end{array}$ & $\begin{array}{l}\text { commettre un crime } \\
\text { prendre la mouche } \\
\text { critique virulente } \\
\text { peur bleue }\end{array}$ \\
\hline 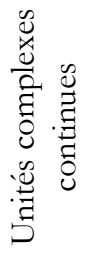 & $\begin{array}{l}\text { Constructions } \\
\text { complexes } \\
\text { morphologiques }\end{array}$ & $\begin{array}{l}\text { Morphosyntaxe et morphologie } \\
\text { [Syntaxe, Lexique] }\end{array}$ & $\begin{array}{l}\text { Morphème du pluriel } \\
\text { portefenille, chou-fleur, } \\
\text { refaire, travailleur }\end{array}$ \\
\hline
\end{tabular}

Tableau 2.

Synthèse à partir de Legallois \& François (2006, p. 15) et Bolly (2008, 2011, p. 33)

Les unités phraséologiques considérées dans leur dimension constructionnelle se distinguent donc à la fois des séquences à combinatoire libre (dont les constituants conservent leur autonomie syntaxique et sémantique respective) et des constructions constituées d'au moins un morphème non autonome (affixe ou désinence). Comme évoqué précédemment, cette gradualité en synchronie (appelée gradience en anglais Traugott \& Trousdale, 2010) peut parfois correspondre à un stade évolutif du processus de grammaticalisation (cf. la notion de gradualness - Traugott \& Trousdale, 2010) des unités linguistiques concernées en diachronie.

En outre, comme le souligne Sinclair (2000, p. 199), les constituants des unités phraséologiques opèrent une co-sélection à la fois sur le plan syntagmatique et paradigmatique. Rompant avec la tradition linguistique, M. Gross $(1975,1982,1988)$ et Halliday $(1966,1985)$ proposaient déjà d'envisager la relation entre le lexique et la grammaire, non plus en termes d'opposition binaire, mais bien en termes d'interdépendance 
lexico-grammaticale: " la lexico-grammaire conçoit notre compétence communicative en termes de cascades de choix : un choix sémiotique, une expression, est réalisé par une série de composants grammaticaux et lexicaux, une construction, et vice versa » (Gledhill et Frath, 2007 : 67). Ce concept de lexique-grammaire nous ramène inévitablement à la notion de valence et, partant, à celle de construction. Par exemple, la différence de valence verbale spécifie deux sens différents de 'prendre' dans $N$ prendre la vie de qqn par comparaison avec $N$ prendre $\varnothing$ vie $\varnothing$ : dans la première structure, le verbe prendre est remplaçable par ôter, la particule de est remplaçable par à et les cases catégorielles (ou slots) du déterminant (ici le dét. défini la) et du complément du nom (dans la structure en $N$ de $N$ ) sont contraintes (le sens véhiculé est celui de 'faire mourir qqn') ; dans la seconde structure avec déterminant et complément nominal «zéro », vie est remplaçable par naissance et les cases catégorielles (ou slots) du déterminant et du complément du nom sont nécessairement vides (le sens véhiculé est celui de 'naître, apparaittre'). Les unités phraséologiques lexico-grammaticales mises au jour en phraséologie statistique (cf. supra) (en particulier les colligations, les patrons, les cadres collocationnels et les collostructions) sont donc en quelque sorte des «structures valencielles à caractère figé » (Bolly, 2011 : 60) au sein desquelles au moins une des cases catégorielles est contrainte lexicalement: "chaque forme lexicale a une lexico-grammaire spécifique, et de même, chaque construction lexico-grammaticale est associée à une série restreinte de formes lexicales » (Halliday, 1966, cité dans Gledhill \& Frath, 2007, p. 66).

\section{Grammaticalisation constructionnelle (approche diachronique)}

On parle actuellement de constructionnalisation pour caractériser le processus de changement langagier impliquant la création d'un nouvel appariement entre une forme et un sens ou une fonction (Traugott, 2008 ; Traugott \& Trousdale, 2010). Dans ce domaine, les études se sont surtout intéressées à l'émergence de structures linguistiques complexes, autrement dit, d'unités lexico-grammaticales telles qu'elles se définissent en phraséologie. Dans leurs travaux, Torres Cacoullos et ses collaborateurs (Torres Cacoullos, 2006, 2011 ; Bybee \& Torres Cacoullos, 2009 ; Torres Cacoullos \& Walker, 2009, 2011) ont ainsi montré que la grammaticalisation portant sur des unités complexes était étroitement liée au phénomène de collocation. À l'instar de ces auteurs, nous pensons que la variation d'emploi de certaines constructions à fonction pragmatique (e.a. le parenthétique $t u$ vois et le marqueur de structuration textuelle on a/nous avons $v u$ que) peut être interprétée comme le reflet d'un processus de grammaticalisation/pragmaticalisation en cours (Bolly, 2010, 2012 ; Bolly \& Degand, soumis). La plurifonctionnalité de la structure [tu+vois] illustre bien cette variation à travers la coexistence d'emplois libres (Ex. 6) et d'emplois conventionnalisés (Ex. 7) en français contemporain.

(6) MARC. Tu trouves que ce tableau n'est pas blanc, Yvan?

YVAN. Pas tout à fait, non...

MARC. Ah bon. Et tu vois quoi comme couleur ?...

YVAN. Je vois des couleurs... Je vois du jaune, du gris, des lignes un peu ocre... (Théâtre, Contemporain, Ext. Frantext- Y. Reza, Art, 1995) 
Flou phraséologique, quasi-grammaticalisation et pseudo marqueurs de discours...

(7) et tu vois en même temps c'est je me dis comme ce ser/ ce serait encore mieux si euh / s'il n'y avait rien eu quoi tu vois /

(Oral, Contemporain, 2004, Valibel, corpus Enquête PFC à Liège, locuteur blaAT11)

Les trois catégories majeures d'emploi de la construction [tu+vois] en français contemporain (constructions régulières, constructions à fonction de commentaire et constructions à fonction pragmatique, cf. infra) peuvent être comprises comme étant chacune représentative d'un stade de pragmaticalisation corrélé à un degré de collocabilité grandissant. Premièrement, les constructions régulières où le verbe a une fonction de matrice verbale (matrix clauses - Dehé \& Wichman, 2010a, 2010b) (Ex. 8) se caractérisent par un faible degré de collocabilité, par leur compositionnalité (avec un faible degré d'abstraction sémantique), ainsi que par la fonction pleinement rectrice du verbe par rapport aux éléments qui l'entourent.

(8) - Tu vois que ça te plaît de te promener. Et la tour Eiffel, tu la vois ? Chichi, lui, il en était toqué. Et les pigeons. Tu en as déjà vu des pigeons ? (Litt., Contemporain, Frantext - R. Forlani, Gouttière, 1989)

D'un point de vue diachronique, si l'on conçoit qu'il y a évolution vers des emplois de plus en plus (inter)subjectifs et de plus en plus autonomes syntaxiquement (cf. Bolly, 2010, 2012), ces constructions avec verbe recteur fort reflèteraient un stade antérieur en grammaticalisation ${ }^{14}$ (Ex. 9).

(9) Oui, j'ai l'air de porter un uniforme blanc. Eh bien! ce n'est pas vrai, c'est faux : je fais semblant! Il frappe sur sa poitrine, sur ses épaules, sur ses bras. Tu vois bien que c'est bleu, que c'est rouge, - regarde ! Colonel ? (Théâtre, Pré-moderne, Frantext - E. Rostand, L'Aiglon, 1900)

Deuxièmement, les constructions à fonction de commentaire (comment clauses cf. Brinton, 2008 ; Dehé \& Wichman, 2010a, 2010b) (Ex. 10) correspondent au stade intermédiaire de l'évolution linguistique en grammaticalisation.

(10) Plus loin, c'est le bruit nouveau des chars de combat. Enfin nous avons entendu des pas et nos yeux se sont ouverts sur la dernière scène. Sanders m’a soufflé : - Tu vois, fallait pas avoir honte. On va brûler gentiment l'un à côté de l'autre. Je l'ai regardé avec horreur. - Après tout, ai-je dit, c'est ça qui me console. (Litt., Moderne, Frantext - R. Nimier, Le Hussard bleu, 1950)

Ces constructions collocationnelles se caractérisent, au niveau formel, par une autonomie syntaxique grandissante de l'unité polylexicale prise dans son ensemble (avec néanmoins une certaine rétention syntaxique). Bien qu'encore partiellement compositionnelles, ces unités se caractérisent par un contenu sémantique plus abstrait (le sens de perception visuelle n'y est plus activé). Troisièmement, à un stade avancé,

\footnotetext{
14 Les études menées en diachronie laissent cependant à penser que le premier stade est virtuel et que les occurrences de tu vois sont dès le départ des constructions à dimension évidentielle (inhérente par ailleurs au sémantisme de voir - cf. Bolly, 2012).
} 
les constructions pragmaticalisées se caractérisent par une autonomie syntaxique et un degré de blanchiment sémantique (ou de non-compositionnalité) maximal par rapport aux stades antérieurs. Il s'agit donc de constructions davantage figées partageant la plupart des traits attribués aux marqueurs pragmatiques (Brinton, 1996) : elles peuvent avoir une fonction discursive interpersonnelle (avec une forte implication de l'interlocuteur dans l'acte d'énonciation), expressive (avec une forte implication du locuteur dans son propre acte d'énonciation) ou de ponctuants (tels que les définissent Dehé \& Wichman, 2010a, 2010b) (Ex. 11).

(11) $[\ldots]$ enfin oui // mouais c'est c'est peut-être parce que / je veux dire / tu vois / moi depuis que je suis à l'université des gens t'en rencontres tout le temps (Oral, Contemporain, 1999, V alibel, corpus Ulco1)

Prenant comme postulat de départ que la grammaticalisation permet à certaines unités lexicales de former de nouvelles collocations (entendues par les auteurs comme étant de facto des unités très figées non analysables) ${ }^{15}$, Torres Cacoullos \& Walker (2011) montrent que les constructions collocationnelles (en l'occurrence, les constructions verbales avec that) sont en fait elles-mêmes graduelles (ce qui a déjà été montré par ailleurs dans les travaux en phraséologie), puisqu'elles conservent des traces de leur fonctionnement syntaxique initial (cf. la notion de retention of linguistic conditioning), ce qui se manifeste entre autres à travers leur potentiel de création/génération syntaxique. Dans une perspective similaire, Van Bogaert (2011) pose que le maintien d'une trace de flexibilité syntaxique au sein des constructions parenthétiques (en l'occurrence, I think) ne permettrait pas de considérer celles-ci comme des marqueurs de discours à part entière dans leur emploi actuel. Notre position sera assurément moins tranchée : ces séquences collocationnelles en cours de constructionnalisation possèdent en effet une fonction spécifiquement discursive, comme c'est le cas par exemple de la construction à fonction d'organisation textuelle nous avons vu (aussi) que (Ex. 12) ou de la construction parenthétique à fonction phatique tu vois (ce que je veux dire) (Ex. 13).

(12) $[\ldots]$ apparemment secondaires, elles remplissent en fait des fonctions essentielles à l'équilibre de l'homme moderne.

Nous avons vu aussi que le loisir ainsi cerné ne se réduit pas à une collection incohérente d'activités. Son contenu présente une structure culturelle. Ces activités [...] (Essai, Moderne, Frantext - N. Bourbaki, Éléments d'histoire des mathématiques, 1966)

(13) L0 et / ça va être sa résidence principale / ou une espèce de maison de campagne?

L1 non ce sera sa résidence principale / oui oui

L0 mmm

L1 et alors tu avais des gens de l'Est qui travaillaient / hein qui chaque fois qu'ils voyaient passer quelqu'un / euh faisaient semblant de siffler ou

\footnotetext{
15 Rien ne permet cependant selon nous de postuler que c'est la grammaticalisation qui provoquerait la conventionnalisation des collocations, et pas l'inverse. C'est aussi la plus grande attraction lexicale entre termes, en même temps que l'augmentation de la fréquence de co-apparition des termes entre eux, qui rend ceux-ci plus collocationnels, donc plus susceptibles de se grammaticaliser (ou d'ailleurs de se lexicaliser ou de se pragmaticaliser).
} 
Flou phraséologique, quasi-grammaticalisation et pseudo marqueurs de discours...

enfin tu vois ce que je veux dire / et qui visiblement se sont fait pincer parce qu'ils ont foutu le camp (Oral, Contemporain, 2004, Valibel, corpus Enquête PFC à Liège, locuteur blaNB1)

Dépassant la vision discrète qui oppose traditionnellement la syntaxe et le discours, nous pensons que ces marqueurs de discours polylexicaux (e.a. les parenthétiques), en tant qu'unités phraséologiques en cours de constructionnalisation, sont des phénomènes graduels fluctuant entre syntaxe et discours. Or, comme le souligne Bybee (2010, p. 6),

$[\mathrm{t}]$ he existence of gradience and variation does not negate the regular patterning within languages or patterning across languages. However, it is important not to view the regularities as primary and the gradience and variation as secondary; rather the same factors operate to produce both regular patterns and the deviations. If language were a fixed mental structure, it would perhaps have discrete categories; but since it is a mental structure that is in constant use and filtered through processing activities that change it, there is variation and gradation.

Partageant à la fois des caractéristiques attribuées aux structures à combinatoire libre et des traits propres aux marqueurs de discours, rien ne permet donc selon nous de catégoriser ces constructions dans l'un ou l'autre domaine langagier de manière univoque.

\section{De la constructionnalisation à la fonctionnalisation}

À côté des mécanismes d'innovation langagière (par ex.: zone piétonne), d'emprunt (par ex. faire son come-back) ou de lexicalisation ${ }^{16}$ (par ex. : porte-manteau, fou rire), les unités phraséologiques qui se conventionnalisent au fil du temps peuvent trouver leur origine dans un changement linguistique impliquant un processus de grammaticalisation (par ex. : se mettre à (rêver, pleurer, etc.), ne... pas, à moins que) (Marchello-Nizia, 2006 ; Torres Cacoullos, 2006, 2011 ; Bybee \& Torres Cacoullos, 2009 ; Torres Cacoullos \& Walker, 2009, 2011) ou de pragmaticalisation (par ex. : prenons l'exemple de..., t'sais, bon appétit.) (Erman \& Kotsinas, 1993 ; Dostie, 2004 ; Bolly, 2010, 2012). Contrairement à Bybee \& Torres Cacoullos (2009) qui envisagent la relation entre phraséologie et grammaticalisation en termes de cause à effet, nous proposons de penser cette relation en termes de corrélation de paramètres (provenant de l'un et l'autre cadre épistémologique) pouvant agir de manière plus large (i) sur le statut grammaticalisé (mais aussi lexicalisé ou pragmaticalisé) de l'unité en question (mono- ou polylexicale) et sur le statut phraséologique de l'unité quand celle-ci est complexe (polylexicale), ou (ii) sur son statut polysémique/plurifonctionnel quand elle est simple (monolexicale). C'est pourquoi, plutôt que le terme de grammaticalisation qui implique une vision du changement où la grammaire prime sur la fonction, mais

16 Dans cet article, l'accent a été mis sur la grammaticalisation et la pragmaticalisation, puisque ces deux processus se sont révélés centraux lors de l'examen des constructions verbales à fonction pragmatique étudiées ici. Pour un examen plus approfondi des relations existant entre grammaticalisation et lexicalisation, nous renvoyons à l'ouvrage de Brinton \& Traugott (2005), ainsi qu'à l'article de Prévost (2006). 
aussi sur le lexique, la sémantique et la pragmatique - nous préférons parler de fonctionnalisation. À noter que dans la tradition non anglo-saxonne, ce pas est sans doute plus aisément franchi, puisque le poids de la tradition générativiste y est moindre. Pour la définir brièvement, la fonctionnalisation se caractériserait par un mécanisme de décatégorialisation allant de pair avec une réanalyse sémantique/pragmatique. Les unités linguistiques susceptibles de subir un processus de fonctionnalisation pourraient ainsi migrer :

(i) du domaine lexical au domaine grammatical (par ex : homo $>$ on) ou du domaine moins grammatical au plus grammatical (par ex. : verbe de mouvement aller $>$ futur périphrastique) [GRAMMATICALISATION (CONSTRUCTIONNELLE)] ;

(ii) du domaine grammatical au domaine lexical (par ex. : un va-nu-pied, un je ne sais quor) [LEXICALISATION (CONSTRUCTIONNELLE)] ;

(iii) du domaine lexical ou grammatical au domaine pragmatique (par ex. : style, genre $>$ connecteur; tu vois, on a vu que $>$ marqueur pragmatique ou textuel) [PRAGMATICALISATION (CONSTRUCTIONNELLE)].

Quant au processus de constructionnalisation, nous proposons de réserver ce terme pour désigner le processus évolutif des unités linguistiques complexes (i.e. évoluant vers un emploi phraséologique), que leur fonction acquise au cours de ce processus de changement soit grammaticale [GRAMMATICALISATION CONSTRUCTIONNELLE], lexicale [LEXICALISATION CONSTRUCTIONNELLE] ou pragmatique [PRAGMATICALISATION CONSTRUCTIONNELLE]. À noter qu'une alternative à ce choix terminologique serait d'opter pour le terme de constructionnalisation comme processus englobant. La notion de construction y serait alors entendue dans son acception large, i.e. comme un appariement conventionnel entre une forme et une fonction sémantique ou discursive susceptible de toucher tous les niveaux de l'analyse langagière : morphèmes, mots, combinaisons syntagmatiques récurrentes, unités phraséologiques collocationnelles ou figées (Goldberg, 2006, p. 5). Cette option impliquerait que la notion de constructionnalisation, essentiellement utilisée à ce jour pour désigner le processus de grammaticalisation affectant des unités linguistiques complexes (cf. supra), soit étendue et redéfinie de manière plus large. Renouant avec les origines fonctionnalistes de la grammaire émergente (usage-based grammar - cf. Bybee, 2010, p. 11), nous pensons pour notre part que le terme de fonctionnalisation est davantage approprié (plus transparent et moins connoté) pour désigner un processus du changement langagier réunissant à la fois la syntaxe, le lexique et le discours, dans une perspective graduelle où les unités tant monolexicales que polylexicales trouveraient leur place.

\section{Conclusion}

Ayant montré en quoi la phraséologie et la grammaticalisation se rejoignaient dans leur conception graduelle des constructions (en synchronie et en diachronie), nous avons vu que l'approche constructionnelle remettait en cause la rupture traditionnellement opérée entre lexique et syntaxe, d'une part, et entre syntaxe et pragmatique, d'autre part. Alors qu'elles ont longtemps été considérées comme étant à 
Flou phraséologique, quasi-grammaticalisation et pseudo marqueurs de discours...

la périphérie de la syntaxe, les unités phraséologiques, définies comme des constructions conventionnalisées, relèvent désormais d'un lexique-grammaire à situer au cœur même du système langagier. Dépassant en outre la vision discrète de l'opposition entre syntaxe et discours, nous avons montré que certaines constructions verbales (en particulier, les marqueurs de discours parenthétiques) peuvent être comprises comme des collocations en cours de pragmaticalisation : ces constructions se caractérisent en effet en français contemporain par un certain degré de collocabilité et possèdent une fonction pragmatique, tout en conservant les traces d'un comportement syntaxique régulier. Enfin, nous avons interrogé la nature du lien existant entre les différents processus du changement langagier (i.e. la lexicalisation, la grammaticalisation, la pragmaticalisation et la constructionnalisation) en proposant d'introduire la notion de fonctionnalisation comme processus évolutif englobant. Cette dernière notion, qui demanderait sans aucun doute à être approfondie, pourra servir (nous l'espérons) de point de départ pour de futures réflexions dans le domaine du changement langagier.

\section{Remerciements}

L'auteur est actuellement chargée de recherches du Fonds de la Recherche Scientifique (F.R.S.-FNRS) de Belgique. Pour mener à bien cette étude, l'auteur a bénéficié d'un subside du Fonds Spécial de Recherche (FSR) de l'Université catholique de Louvain et d'un crédit du F.R.S.-FNRS pour bref séjour à l'Université de Neuchâtel (Suisse). Cette étude s'intègre par ailleurs dans le Pôle d'Attraction Interuniversitaire «Grammaticalization and (inter)Subjectification » (contrat PAI P6/44) financé par le gouvernement fédéral belge.

Je tiens à remercier les relecteurs anonymes dont les commentaires précieux ont permis d'améliorer considérablement la qualité du texte original. Je remercie en particulier Sophie Prévost, Marie-José Béguelin, Britt Erman et Elizabeth Traugott pour les échanges cordiaux que nous avons eus ces dernières années et qui ont profondément nourri ma réflexion sur l'interface phraséologie/grammaticalisation. 


\section{RÉFÉRENCES}

Altenberg, B. (1998), «On the phraseology of spoken English : The evidence of recurrent word-combinations", in A. P. Cowie (ed.), Phraseology: Theory, Analysis, and Applications, Oxford, Oxford University Press, p. 101-122.

BALLy, C. (1909), Traité de stylistique française, Paris, Klincksieck.

BÉGUELIN, M.-J. (2009), «From the confession of ignorance to the indefinite: What impact for a theory of grammaticalization ?", in C. Rossari, C. Ricci \& A. Spiridon (eds.), Facts, Approaches, Theoretical Issues, Bingley, Emerald (Studies in Pragmatics), p. 35-64.

BÉGuELIN, M.-J. (2010), "Le statut des identités diachroniques dans la théorie saussurienne: une critique anticipée du concept de grammaticalisation », in J.-P. Bronckart, C. Bota \& E. Bulea (eds.), Le projet de Saussure, Genève, Droz, p. 237-268.

BIBER, D., CONRAD, S. \& CORTES, V. (2004), «If you look at... : Lexical bundles in University teaching and textbooks », Applied Linguistics, $n^{\circ} 25$, p. 371-405.

Blanc, O., Constant, M., Dister, A. \& Watrin, P. (2008), "Corpus oraux et chunking », 27èmes Journées d'Études sur la Parole (JEP'08).

Blanc, O., Constant, M., Dister, A. \& Watrin, P. (2010), «Partial parsing of spontaneous spoken French», 7th International Conference on Language Resources and Evaluation (LREC'10), May 2010.

BOLly, C. (2008), Les unités phraséologiques : un phénomène linguistique complexe? (Thèse de doctorat non publiée), Louvain-la-Neuve, Université catholique de Louvain.

Bolly, C. (2010), «Pragmaticalisation du marqueur discursif 'tu vois'. De la perception à l'évidence et de l'évidence au discours ", in F. Neveu, J. Durand, T. Klingler, S. Prévost \& V. Muni-Toké (eds.), Actes du CMLF 2010 (2 Congrès Mondial de Linguistique Française, 12-15 juillet 2010, New Orleans, Etats-Unis), http://dx.doi.org/10.1051/cmlf/2010243.

BOLly, C. (2011), Phraséologie et collocations. Approche sur corpus en français L1 et L2, Bruxelles, Bern, Berlin, Frankfurt am Main, New York, Oxford, Wien, P.I.E. Peter Lang (GRAMM-R. Études de linguistique française 9).

Bolly, C (2012), «Du verbe de perception visuelle au marqueur parenthétique 'tu vois': Grammaticalisation et changement linguistique », Journal of French Language Studies, n²2(2), p. 143-164.

Bolly, C. \& Degand, L., (sous presse), «Have you seen what I mean? From verbal constructions to discourse structuring markers », Journal of Historical Pragmatics.

BRINTON, L. J. (1996), Pragmatic markers in English: Grammaticalization and discourse functions, Berlin, Mouton de Gruyter.

BRINTON, L. J., (2008), The comment clause in English. Syntactic origins and pragmatic development, Cambridge, Cambridge University Press (Studies in English Language).

BRINTON, L. J. \& TRAugOTT, E. C. (2005), Lexicalization and language change, Cambridge, Cambridge University Press. 
Flou phraséologique, quasi-grammaticalisation et pseudo marqueurs de discours...

BYBEE, J. (2003), "Mechanisms of change in grammaticalization: The role of frequency », in B. D. Joseph \& R. D. Janda (eds.), The handbook of historical linguistics, Oxford, Blackwell, p. 604-623.

BYBEe, J. (2010), Language, usage and cognition, Cambridge, Cambridge University Press.

Bybee, J. \& Torres Cacoullos, R. (2009), "The role of prefabs in grammaticization: How the particular and the general interact in language change», in R. Corrigan, E. Moravcsik, M. Oulali \& K. Wheatley (eds.), Formulaic language (Volume 1 : Distribution and historical change), Amsterdam, Philadelphia, John Benjamins (Typological Studies in Language 82), p. 187-218.

CAmpbell, L. (2001), "What's wrong with grammaticalization ?", Language Sciences, $\mathrm{n}^{\circ} 23$, p. 113-162.

CONKLIN, K. \& SCHMITT, N. (2008), «Formulaic sequences : Are they processed more quickly than nonformulaic language by native and nonnative speakers? ", Applied Linguistics, $\mathrm{n}^{\circ} 29(1)$, p. $72-89$.

CowIE, A. P. (ed.) (1998), Phraseology : Theory, analysis, and applications, Oxford, Oxford University Press.

Croft, W. (1990), Typology and Universals, Cambridge, Cambridge University Press.

DE COCK, S. (2003), Recurrent sequences of words in native speaker and advanced learner spoken and written English: A corpus-driven approach (Thèse de doctorat non publiée), Louvain-laNeuve, Université catholique de Louvain.

DE COCK, S. (2004), « Preferred sequences of words in NS and NNS speech », Belgian Journal of English Language and Literatures, New Series $\mathrm{n}^{\circ} 2$, p. 225-246.

Dehé, N. \& Wichmann, A. (2010a), «Sentence-initial I think (that) and I believe (that) : Prosodic evidence for uses as main clause, comment clause and discourse marker ", Studies in Language, $\mathrm{n}^{\circ} 34(1)$, p. 36-74.

Dehé, N. \& Wichmann, A. (2010b), "The multifunctionality of epistemic parentheticals in discourse : Prosodic cues to the semantic-pragmatic boundary ", Functions of Language, $\mathrm{n}^{\circ} 17(1)$, p. 1-28.

De Mulder, W. (2001), « La linguistique diachronique, les études sur la grammaticalisation et la sémantique du prototype : Présentation », Langue française, $\mathrm{n}^{\circ} 130(1)$, p. 8-32.

DIEWALD, G. (2006), «Context types in grammaticalization as constructions », in D. Schönefeld (ed.), Constructions. Special Volume 1 : Constructions all over - case studies and theoretical implications, http://www.constructions-online.de/articles/specvol1/.

Dostie, G. (2004), Pragmaticalisation et marqueurs discursifs. Analyse sémantique et traitement lexicographique, Bruxelles, De Boeck-Duculot (Champs linguistiques).

ElLis, N. C., Simpson-Vlach, R. \& MAYNARD, C. (2008), «Formulaic language in native and second-language speakers: Psycholinguistics, corpus linguistics, and TESOL», Tesol Quarterly, n42, p. 375-396.

ERMan, B. \& Kotsinas, U.-B. (1993), «Pragmaticalization : the case of 'ba' and 'you know'», Studier i modern språkvetenskap, n¹0, p. 76-93. 
ERMAN, B. \& WARREN, B. (2000), «The idiom principle and the open choice principle », Tekst, $\mathrm{n}^{\circ} 20(1)$, p. 29-62.

Fiala, P., LAfon, P. \& Piguet, M.-F. (eds.) (1997), La locution: Entre lexique, syntaxe et pragmatique, Paris, Klincksieck (Collection Saint-Cloud).

FIRTH, J. R. (1957), «A synopsis of Linguistic Theory 1930-1955 », Transactions of the Philological Society (Special Volume) (= Studies in Linguistic Analysis), p. 1-32. Ré-imprimé in F. R. Palmer (ed.), 1968, Selected Papers of J.R. Firth 1952-1959, London, Longman.

FORSBERG, F. (2008), Le langage préfabriqué. Formes, fonctions et fréquences en français parlé L2 et L1, Bern-Berlin, Peter Lang (Contemporary studies in descriptive linguistics 20).

Francis, G. (1993), «A corpus-driven approach to grammar: Principles, methods and examples », in M. Baker, G. Francis \& E. Tognini-Bonelli (eds.), Text and Technology : In Honour of John Sinclair, Amsterdam, Philadelphia, John Benjamins, p. 137-156.

FrAnÇOIS, J. (ed.) (2008), «Les Grammaires de construction, un bâtiment ouvert aux quatre vents », Cabiers du Crisco, n²6, http://www.crisco.unicaen.fr/Les-grammaires-deconstruction.html.

François, J. \& MANGuin, J.-L. (2006), «Dispute théologique, discussion oiseuse et conversation téléphonique : Les collocations adjectivo-nominales au cœur du débat», in P. Blumenthal \& F. J. Hausmann (ed.), Collocations, corpus, dictionnaires (= Langue française, $\mathrm{n}^{\circ} 150$ ), p. 50-65.

FuCHS, C. (2004), «Pour introduire à la linguistique cognitive», in C. Fuchs, La linguistique cognitive, Paris, Ophrys, http://halshs.archives-ouvertes.fr/halshs-00067934/fr/.

Gledhill, C. \& Frath, P. (2007), «Collocation, phrasème, dénomination : Vers une théorie de la créativité phraséologique », La Linguistique, $n^{\circ} 43$, p. 63-88.

GOldBerg, A. (2006), Constructions at work: The nature of generalization in language, Oxford, Oxford University Press.

GonzÁlez Rey, I. (2002), La phraséologie du français, Toulouse, Presses universitaires du Mirail.

Granger, S. \& PAQUOT, M. (2008a), «From dictionary to phrasebook? », in E. Bernal \& J. DeCesaris (eds.), Proceedings of the XIII EURALEX International Congress, Barcelona, 15-19 July 2008, p. 1345-1355.

Granger, S. \& PAQUot, M. (2008b), « Disentangling the phraseological web », in S. Granger \& F. Meunier (eds.), Phraseology: An interdisciplinary perspective, Amsterdam, Philadelphia, John Benjamins, p. 27-49.

GRIES, S. T. \& STEFANOWITSCH, A. (2004), «Extending collostructional analysis. A corpusbased perspective on 'alternations'», International Journal of Corpus Linguistics, $\mathrm{n}^{\circ} 9(1)$, p. 97-129.

Gross, G. (1996), Les expressions figées en français. Noms composés et autres locutions, Paris, Ophrys.

Gross, M. (1975), Méthodes en syntaxe, Paris, Hermann.

Gross M. (1982), «Une classification des phrases 'figées' du français», Revue Québécoise de linguistique, $\mathrm{n}^{\circ} 11(2)$, p. 151-185.

Gross, M. (1988), «Les limites de la phrase figée », Langages, nº9, p. 7-22.

Habert, B., Nazarenko, A. \& Salem, A. (1997), Les linguistiques de corpus, Paris, Armand Colin. 
Flou phraséologique, quasi-grammaticalisation et pseudo marqueurs de discours...

Halliday, M. A. K. (1966), "Lexis as a linguistic level», in C. Bazell, J. Catford, M. A. K. Halliday \& R. Robins (eds.), In Memory of J. R. Firth, London, Longman, p. 148162.

Halliday, M. A. K. (1985), An introduction to functional grammar, London, Arnold.

Haspelmath, M. (2004), "On directionality in language change with particular reference to grammaticalization », in O. Fischer, M. Norde \& H. Perridon (eds.), Up and down the cline : The nature of grammaticalization, Amsterdam, John Benjamins, p. 17-44.

HeIne, B. (1993), Auxiliaries: Cognitive forces and grammaticalization, Oxford, Oxford University Press.

HeINE, B. (2002), « On the role of context in grammaticalization », in I. Wischer \& G. Diewald (eds.), New reflections on grammaticalization, Amsterdam, Philadelphia, John Benjamins (Typological studies in language 49), p. 83-101.

HeINE, B. (2003), «Grammaticalization», in B. D. Joseph \& R. D. Janda (eds.), Handbook of historical linguistics, Oxford, Blackwell, p. 575-601.

HoEY, M. (2000), «A world beyond collocation : New perspectives on vocabulary teaching », in M. Lewis (ed.), Teaching collocation. Further developments in the lexical approach, Hove, Language Teaching Publications, p. 224-243.

Hopper, P. J. (1987), «Emergent Grammar », Berkeley Linguistics Conference (BLS), n¹3, p. 139157.

Hopper, P. J. (1991), «On some principles of grammaticalization», in E.C. Traugott \& B. Heine, (eds.), Approaches to grammaticalization (2 vol.), Amsterdam, Philadelphia, John Benjamins, p. 17-35.

Hopper, P. J. (1998), «Emergent Grammar», in M. Tomasello (ed.), The new psychology of language, Mahwah, Lawrence Erlbaum Associates, p. 155-175.

Hunston, S. \& Francis, G. (2000), Pattern grammar. A corpus-driven approach to the lexical grammar of English, Amsterdam, Philadelphia, John Benjamins.

HYLAND, K. (2008), «As can be seen : Lexical bundles and disciplinary variation", English for Specific Purposes, n²7, p. 4-10.

LAMiroY, B. \& KLEIN, J. R. (2005), « Le problème central du figement est le semi-figement », in A. Balibar-Mrabti \& C. Vaguer (eds.), Le semi-figement (= LINX, n53), p. 135-154.

LANGACKeR, R. W. (2000), "A dynamic usage-based model», in M. Barlow \& S. Kemmer (eds.), Usage-based models of language, Stanford, CSLI, p. 1-63.

LEgallois, D. \& FrançOIS, J. (2006), «Autour des grammaires de construction et de patterns », Cabier du Crisco, n²1, http://elsap1.unicaen.fr/cahiers/.

LEGALLOIS, D. \& GRÉA, P. (2007), «L'objectif de cet article est de... : Construction spécificationnelle et grammaire phraséologique ", Cabiers de praxématique, n46, p. 161-184.

LEHMANN, C. (1995), Thoughts on grammaticalization, Munich, LINCOM-Europa.

MARCHELlo-NizIA, C. (2006), Grammaticalisation et changement linguistique, Bruxelles, De BoeckDuculot (Champs linguistiques). 
MARTins-BALTAR, M. (ed.) (1995), La locution en discours (= Cabiers du français contemporain, $\mathrm{n}^{\circ} 2$ ), Paris, Didier érudition.

Martins-BAltar, M. (ed.) (1997), La locution entre langue et usages, Paris, ENS éditions Fontenay/Saint-Cloud (Signes), diffusion Ophrys.

McEnery, T. \& Wilson, A. (1996), (2001) (2e éd.), Corpus linguistics, Edinburgh, Edinburgh University Press.

MeILLET A. (1913), "Sur la méthode de la grammaire comparée », in Revue de métaphysique et de morale $\mathrm{n}^{\circ} 21(1)$, p. 1-15, repris dans Linguistique historique et linguistique générale, 1948, Paris, Honoré Champion, p. 19-35.

MEJRI, S. (1997), Le figement lexical. Descriptions linguistiques et structuration sémantique, Tunis, Publications de la faculté des lettres de la Manouba.

MejRI, S. (2005), «Figement absolu ou relatif: La notion de degré de figement », Le Semifigement (= LINX, n53), p. 183-196.

MEL'ČUK, I. (1993), « La phraséologie et son rôle dans l'enseignement/apprentissage d'une langue étrangère », Revue de Linguistique appliquée, n 92 , p. 82-113.

MEL'ČUK, I. (1995), « Phrasemes in Language and Phraseology in Linguistics », in M. Everaert et al. (eds.), Idioms : Structural and psychological perspectives, Hillsdale, Lawrence Erlbaum Associates, p. $167-232$.

Mel’ČUK, I. (1998), "Collocations and Lexical Functions », in A. P. Cowie (ed.), Phraseology. Theory, analysis, and applications, Oxford, Oxford University Press, p. 23-53.

MOON, R. (1998), Fixed expressions and idioms in English, Oxford, Clarendon Press.

NEWMEYER, F. J. (2001), «Deconstructing grammaticalization», Language Sciences, n²3, p. 187230.

Norde, M. (2010), «Degrammaticalization: Three common controversies », in K. Stathi, E. Gehweiler \& E. König (eds.), Grammaticalization. Current views and issues, Amsterdam, Philadelphia, John Benjamins, p. 123-150.

PrÉvost, S. (2003), «La grammaticalisation : Unidirectionnalité et statut », Le Français Moderne, n71(2), p. 144-166.

PRÉVOST, S. (2006), « Grammaticalisation, lexicalisation et dégrammaticalisation : Des relations complexes », Cahiers de praxématique, n 46 , p. 121-139.

RAstier, F. (2005), « La microsémantique », Texto!, http:/ /www.revue-texto.net/index.php?id=536.

Rastier, F., Cavazza, M. \& Abeillé, A. (1994), Sémantique pour l'analyse. De la linguistique à l'informatique, Paris, Masson.

RÖMER, U. (2009), "The inseparability of lexis and grammar : Corpus linguistic perspectives », Annual Review of Cognitive Linguistics, $\mathrm{n}^{\circ}$, p. 141-163.

SCHNEIDER, S. (2007), Reduced parenthetical clauses as mitigators : A corpus study in Spoken French, Italian and Spanish, Amsterdam, Philadelphia, John Benjamins.

SCOTT, M. (2004-2007), Oxford WordSmith Tools 4.0., logiciel informatique, http://www.lexically.net/ downloads/version4/wordsmith.pdf. 
Flou phraséologique, quasi-grammaticalisation et pseudo marqueurs de discours...

SinClaIR, J. (1991), Corpus, concordance, collocation, Oxford, Oxford University Press.

SINCLAIR, J. (2000), «Lexical grammar», Nanjoji Metodologija, n²4, p. 191-203, http://donelaitis.vdu.lt/ publikacijos/sinclair.pdf.

SinClAir, J. \& ReNOUf, A. (1991), "Collocational frameworks in English», in K. Aijmer \& B. Altenberg (eds.), English corpus linguistics: Studies in honour of Jan Svartvik, Amsterdam, Philadephia, John Benjamins, p. 128-144.

Stefanowitsch, A. \& GRIES, S. T. (2003), «Collostructions : Investigating the interaction of words and constructions », International Journal of Corpus Linguistics, n 8 (2), p. 209-243.

STUBBS, M. (1995), «Collocations and semantic profiles: On the cause of the trouble with quantitative studies », Functions of Language, n²2(1), p. 23-55.

Svensson, M. H. (2004), Critères de figement. L'identification des expressions figées en français contemporain, Umeå university, Institutionen för Moderna Språk Publikationer.

TORRES CaCoullos, R. (2006), "Relative frequency in the grammaticization of collocations : Nominal to concessive a pesar de », in T. L. Face \& C. A. Klee (eds.), Selected proceedings of the 8th Hispanic Linguistics Symposium, Somerville, Cascadilla Proceedings Project, p. 37-49.

TORRES CaCoullos, R. (2011), «Variation and grammaticalization », in M. Díaz-Campos (ed.), The handbook of hispanic sociolinguistics, Oxford, Wiley-Blackwell, doi : 10.1002/9781444393446.ch7.

Torres Cacoullos, R. \& Walker, J. A. (2009), « On the persistence of grammar in discourse formulas : A variationist study of that», Linguistics, $\mathrm{n}^{\circ} 47$, p. 1-43.

TORres CaCoullos, R. \& WALKer, J. A. (2011), «Collocations in grammaticalization and variation ", in B. Heine \& H. Narrog (eds.), The Oxford handbook of grammaticalization (Chapter 18), Oxford, Oxford University Press.

TraugotT, E. C. (1982), «From propositional to textual and expressive meanings : Some semantic-pragmatic aspects of grammaticalization », in W. P. Lehmann \& Y. Malkiel (eds.), Perspectives on historical linguistics, Amsterdam, John Benjamins, p. 245-271.

TRAugotT, E. C. (1997), "The role of the development of discourse markers in a theory of grammaticalization », communication à ICHL XII, Manchester 1995 (version du 11/97).

TRAUGOTT, E. C. (2001), «Legitimate counterexamples to unidirectionality », article présenté à l'Université de Freiburg, le 17 octobre 2001.

TraugotT, E. C. (2008), “'All that he endeavored to prove was...': On the ermergence of grammatical constructions in dialogual and dialogic contexts", in R. Kempson \& R. Cooper (eds.), Language in flux: Dialogue coordination, language variation, change and evolution, London, Kings College Publications.

TraugotT, E. C. (2010), " (Inter)subjectivity and (inter)subjectification : A reassessment », in H. Cuyckens, K. Davidse \& L. Vandelanotte (eds.), Subjectification, intersubjectification and grammaticalization, Berlin, New York, Mouton de Gruyter, p. 29-74.

Traugott, E. C. \& Trousdale, G. (2010), « Gradience, gradualness, and grammaticalization : How do they intersect? », in E. C. Traugott \& G. Trousdale (eds.), Gradience, gradualness, and grammaticalization, Amsterdam, Philadelphia, John Benjamins, p. 19-44. 
Catherine Bolly

TutiN, A. (2010), «Les collocations dans les dictionnaires monolingues spécialisés de collocations », in F. Neveu, J. Durand, T. Klingler, S. Prévost \& V. Muni-Toké (eds.), Actes du CMLF 2010 (2e Congrès Mondial de Linguistique Française, $12-15$ juillet 2010, New Orleans, EtatsUnis), http://dx.doi.org/10.1051/cmlf/2010141.

Urmson, J. (1952), « Parenthetical verbs », Mind, nº61, p. 480-496.

VAN BOGAERT, J. (2011), «I think and other complement-taking mental predicates: A case of and for constructional grammaticalization », Linguistics, n 49 (2), p. 295-332.

Wood, D. (ed.) (2010), Perspectives on formulaic language : Acquisition and communication, London, New York, Continuum.

Wray, A. (2002), Formulaic language and the lexicon, Cambridge, Cambridge University Press.

WraY, A. (2007), " 'Needs only' analysis in linguistic ontogeny and phylogeny", in C. Lyon, C. L. Nehaniv \& A. Cangelosi (eds.), Emergence of communication and language (Part 1), London, Springer Verlag, p. 53-70.

WRAY, A. (2008), Formulaic language : Pushing the boundaries, Oxford, Oxford University Press.

\section{Corpus}

ComparaF [Corpus équilibré en français de France et de Belgique] = Bolly, C., (2008), Les unités phraséologiques: un phénomène linguistique complexe? (Thèse de doctorat non publiée), Louvain-la-Neuve, Université catholique de Louvain.

Valibel $=$ Dister, A., Francard, M., Hambye, P. \& Simon, A.C., (2009), « Du corpus à la banque de données. Du son, des textes et des métadonnées. L'évolution de banque de données textuelles orales VALIBEL (1989-2009)», Cabiers de Linguistique, n³3(2), p. 113-129, http://www.uclouvain.be/valibel. 